УДК 624.072.33

\title{
ВЛИЯНИЕ ПАРАМЕТРОВ ПРОЕКТИРОВАНИЯ НА ОПТИМАЛЬНОСТЬ КОНСТРУКЦИИ ТРЕХШАРНИРНЫХ АРОК
}

Канд. техн. наук Г.Л. Ватуля, канд. техн. наук Е.Ф. Орел, С.В. Левчук, И.М. Андрущенко

\section{ВПЛИВ ПАРАМЕТРІВ ПРОЕКТУВАННЯ НА ОПТИМАЛЬНІСТЬ КОНСТРУКЩІЇ ТРИШАРНІРНИХ АРОК}

Канд. техн. наук Г.Л. Ватуля, канд. техн. наук С.Ф. Орел, С.В. Левчук, І.М. Андрущенко

\section{IMPACT OF DESIGN PARAMETERS ON THREE-HINGED ARCH STRUCTURAL OPTIMIZATION}

Cand. of techn. sciences G.L. Vatulya, cand. of techn. sciences E.F. Orel, S.V. Levchyk, S.M. Andrushenko

Получены выражения, позволяющие оченить влияние параметров проектирования на выбор оптимальной конструкции трехшарнирной арки прямоугольного поперечного сечения. Оптимальный проект сравнивается с аркой постоянного сечения.

Ключевые слова: целевая функиия, степень равнопрочности, оптимальное поперечное сечение, переменные параметры, условие прочности, сложная деформация.

Отримано математичні вирази, щзо дозволяють оцінити вплив параметрів проектування на вибір оптимальної конструкції тришарнірної арки прямокутного поперечного перерізу. Оптимальний проект порівнювався із аркою постійного перерізу.

Ключові слова: цілььова функиія, ступінь рівноміцності, оптимальний поперечний переріз, змінні параметри, умова міиності, складна деформація.

In the article authors obtain the mathematical functions allows to provide the design parameters evaluation on rectangular three-hinged arch structure optimality. The optimal structures were matched with uniform cross-section arch.

Key words: efficiency function, firmness degree, optimum cross section, variable parameters, strength condition, complex deformation.

Введение. Проектирование на стадии создания технического проекта связано с конструктивными разработками элементов узлов и соединений, с производством расчетов для определения размеров элементов и их сечений и создания рабочих чертежей. Сооружение, построенное по оптимальному проекту, должно отличаться от подобных других меньшей затратой материала, меньшей массой и стоимостью.
Цель исследований. Статически определимые трехшарнирные арки. Каждое подмножество систем описывается присущими ему параметрами, которые зависят от распределения усилий. Усилия определяются только из уравнений равновесия или совместно с уравнениями деформаций $[1,2]$. Уравнения равновесия учитывают геометрию системы, а уравнения неразрывности деформаций еще и влияние распределения параметров. 
Таким образом, оптимальность проекта зависит от многих факторов и различной степени их влияния [3]. Систематизация этого процесса поможет более эффективно использовать идею оптимальности при проектировании конструкций.

Основной материал исследований

1.Трехшарнирная арка постоянного сечения. Определив реактивные силы, рассмотрим круговую арку (рисунок) пролетом $\ell=30 \mathrm{~m}$ прямоугольного поперечного сечения. Арка находится под воздействием постоянной нагрузки $q=6 \mathrm{Tc} / \mathrm{M}$, расчетное сопротивление $m R_{y}=2000 \kappa г \mathrm{~cm}{ }^{2}$. Внутренние усилия в произвольном сечении будут равны:

$$
\begin{gathered}
M_{x}=\frac{q x}{2}(\ell-x)-\frac{q \ell^{2}}{8 f} y, \\
Q_{x}=\frac{q(\ell-2 x)}{2} \cos \varphi-\frac{q \ell^{2}}{8 f} \sin \varphi, \\
N_{x}=\frac{q(\ell-2 x)}{2} \sin \varphi-\frac{q \ell^{2}}{8 f} \cos \varphi .
\end{gathered}
$$

Условие прочности по нормальным напряжениям запишем в виде

$$
Q_{\max }=\frac{M_{x}}{W}+\frac{N_{x}}{A} \leq R_{y} .
$$

Уравнение оси круговой арки

$$
y=\frac{1}{2}\left[\sqrt{4 R^{2}-\ell^{2}+4 x(\ell-x)}-\sqrt{4 R^{2}-\ell^{2}}\right] .
$$

Стрела подъема арки

$$
f=R-\frac{1}{2} \sqrt{4 R^{2}-\ell^{2}} .
$$

Она при заданном пролете может изменяться в зависимости от величины радиуса кривой в пределах (от $R=\ell / 2$ до $R=\ell$ )

$$
\frac{\ell}{2} \geq f \geq \frac{\ell}{2}(2-\sqrt{3})
$$

Используем выражения для тригонометрических функций:

Тогда

$$
\begin{aligned}
& \sin \varphi= \frac{\ell-2 x}{2 R}, \\
& \cos \varphi=\frac{y+\frac{1}{2} \sqrt{4 R^{2}-\ell^{2}}}{R}=\frac{\sqrt{4 R^{2}-\ell^{2}+4 x(\ell-x)}}{2 R} .
\end{aligned}
$$

$$
\begin{gathered}
M_{x}=\frac{q x(\ell-x)}{2}-\frac{q \ell^{2}}{8}\left[\frac{\sqrt{4 R^{2}-\ell^{2}+4 x(\ell-x)}-\sqrt{4 R^{2}-\ell^{2}}}{2 R-\sqrt{4 R^{2}-\ell^{2}}}\right], \\
Q_{x}=\frac{q(\ell-2 x)}{16 f R}\left[4 f \sqrt{4 R^{2}-\ell^{2}+4 x(\ell-x)}-\ell^{2}\right], \\
N_{x}=\frac{q \ell^{2}}{4 R}\left[\frac{(\ell-2 x)^{2}}{\ell^{2}}+\frac{\sqrt{4 R^{2}-\ell^{2}+4 x(\ell-x)}}{2\left(2 R-\sqrt{4 R^{2}-\ell^{2}}\right)}\right] .
\end{gathered}
$$


a)

б)

B)

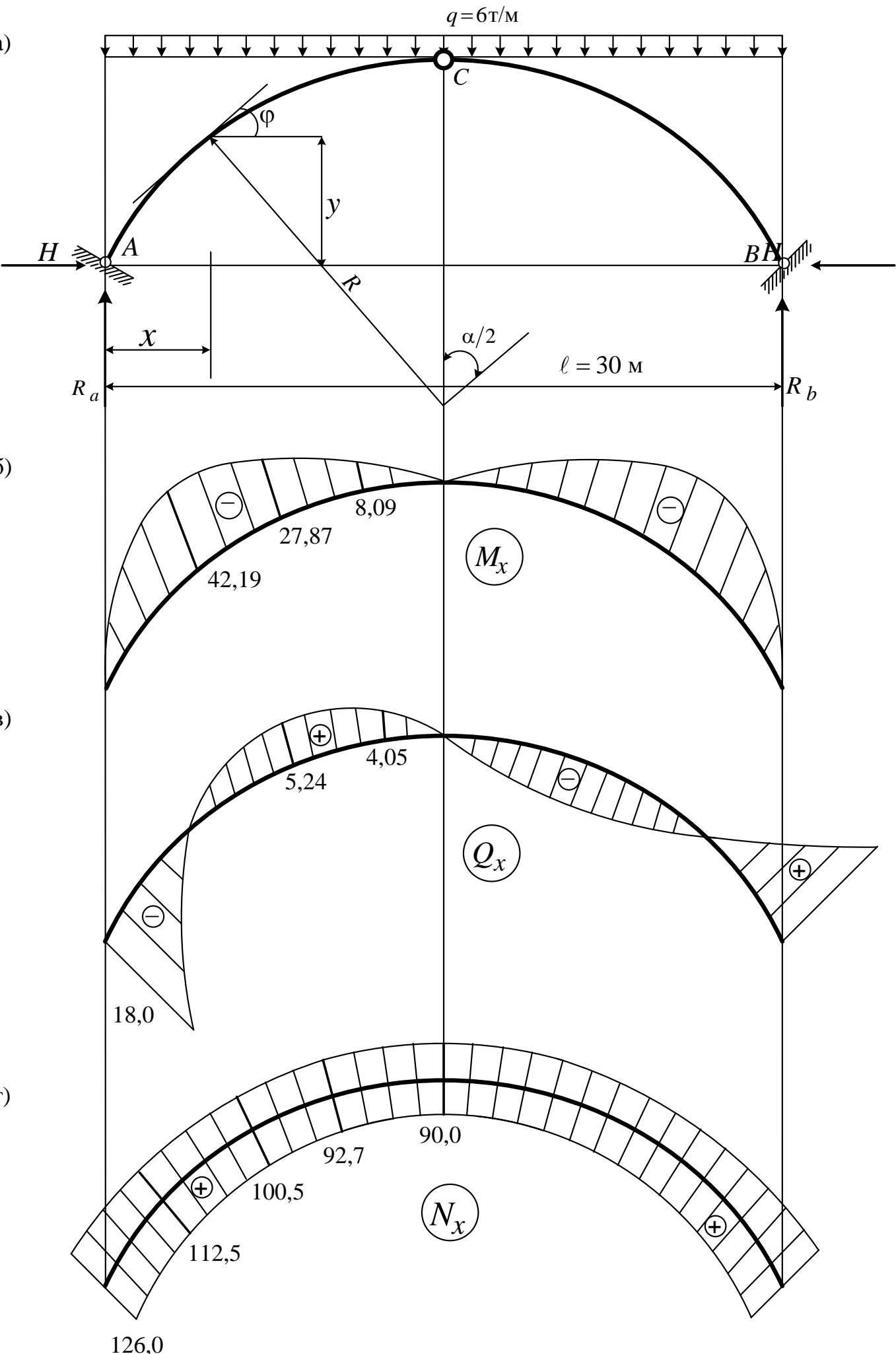

Рис. 1. Расчетная схема трехшарнирной арки 
Рассмотрим частный случай, когда $R=\frac{5 \ell}{8}$, т.е.

$$
\begin{gathered}
M_{x}=\frac{q \ell^{2}}{2}\left[\frac{x}{\ell}\left(1-\frac{x}{\ell}\right)-\sqrt{\frac{9}{64}+\frac{x}{\ell}\left(1-\frac{x}{\ell}\right)}+\frac{3}{8}\right], \\
Q_{x}=\frac{2 q \ell}{5}\left(1-\frac{2 x}{\ell}\right)\left[2 \sqrt{\frac{9}{64}}+\frac{x}{\ell}\left(1-\frac{x}{\ell}\right)-1\right], \\
N_{x}=\frac{2 q \ell}{5}\left[\left(1-\frac{2 x}{\ell}\right)^{2}+2 \sqrt{\frac{9}{64}}+\frac{x}{\ell}\left(1-\frac{x}{\ell}\right)\right] .
\end{gathered}
$$

Эпюры полученных внутренних усилий показаны на рисунке.

Сечения арки испытывают сложную деформацию - изгиб с сжатием. Условие прочности по нормальным напряжениям в этом случае имеет вид

$$
\sigma_{\max }=\frac{|M|}{W}+\frac{N}{A} \leq m R_{y} .
$$

В рассматриваемом напряженном состоянии напряжения зависят от изгибающего момента и продольной силы, но наибольшее влияние на их величину оказывает изгибающий момент. Поэтому найдем сечение, в котором изгибающий момент достигает максимального значения, т.е. где

$$
\frac{d M_{x}}{d x}=0
$$

Обозначим $\frac{x}{\ell}=\xi$, тогда

$$
\sigma_{\max }=\frac{\left|-42,19 \cdot 10^{5}\right|}{12 \cdot 35^{2}} \cdot 6+\frac{112,5}{12 \times 35}=1722+268=1990 \approx 2000 \kappa г \mathrm{c} / \mathrm{cм}^{2}
$$

Затрата материала на арку постоянного сечения 


$$
\begin{gathered}
V_{\text {п }}=b h \ell_{\mathrm{a}}=b h \frac{2 \pi R}{360} \cdot \alpha=b h \frac{\pi R}{180} 2 \operatorname{arctg} \frac{\ell}{2(R-f)}=12 \cdot 35 \frac{\pi \cdot 18,75 \cdot 10^{2}}{90} \times \\
\times \operatorname{arctg} \frac{30}{2(18,75-7,5)}=1460500 \mathrm{~cm}^{3} .
\end{gathered}
$$

2. Непрерывно равнопрочная трехшарнирная арка. В такой арке условие равнопрочности в каждом сечении выполняется в виде равенства. Рассмотрим арку с заданной шириной сечения $b=12 \mathrm{~cm}$ и переменной высотой $h_{x}$. Запишем условие прочности для произвольного сечения арки:

$$
\sigma_{x}=\frac{6\left|M_{x}\right|}{b h_{x}^{2}}+\frac{N_{x}}{b h_{x}}=m R_{y}
$$

Отсюда получаем уравнение для определения функции высоты сечения $h_{x}$

$$
h_{x}^{2}-\frac{N_{x}}{b m R_{y}}-\frac{6 M_{x}}{b m R_{y}}=0
$$

Решая уравнение (12), получаем действительный корень

$$
h_{x}=\frac{N_{x}}{2 b m R_{y}}+\sqrt{\left(\frac{N_{x}}{2 b m R_{y}}\right)^{2}+\frac{6\left|M_{x}\right|}{b m R_{y}}}
$$

Подставив выражения для $M_{x}$ и $N_{x}$, получаем функцию изменения высоты сечения арки вдоль ее оси

$$
\begin{aligned}
& h_{x}=\frac{q \ell}{5 b R_{y}}\left[\left(1-\frac{2 x}{\ell}\right)^{2}+2 \sqrt{\frac{9}{64}+\frac{x}{\ell}\left(1-\frac{x}{\ell}\right)}\right]+ \\
& +\sqrt[\left\{\frac{q \ell}{5 b R_{y}}\left[\left(1-\frac{2 x}{\ell}\right)^{2}+2 \sqrt{\frac{9}{64}+\frac{x}{\ell}\left(1-\frac{x}{\ell}\right)}\right]\right\}^{2}+]{+\frac{3 q \ell^{2}}{b R_{y}}\left[\frac{x}{\ell}\left(1-\frac{x}{\ell}\right)-\sqrt{\frac{9}{64}+\frac{x}{\ell}\left(1-\frac{x}{\ell}\right)}+\frac{3}{8}\right]} .
\end{aligned}
$$

Высоты сечений приведены в таблице.

Таблица

Высота сечения арки вдоль ее оси

\begin{tabular}{|c|c|c|c|c|c|}
\hline$x / \ell$ & 0 & $1 / 8$ & $1 / 4$ & $3 / 8$ & $1 / 2$ \\
\hline$h_{x}, \mathrm{~cm}$ & 4,8 & 34,9 & 28,6 & 16,3 & 3,4 \\
\hline
\end{tabular}

Теоретический объем материала равнопрочной арки $V_{p}=1010000 \mathrm{~cm}^{3}$.
На самом деле объем будет несколько большим за счет увеличения высоты 
приопорных сечений для восприятия сдвигающих усилий.

Выводы. Таким образом, получены выражения, позволяющие оценить влияние параметров проектирования на выбор оптимальной конструкции трехшарнирной арки. Уменьшение объема по сравнению с аркой постоянного сечения достигается на $30 \%$.

\section{Список использованных источников}

1. Виноградов, А.И. Проблема оптимального проектирования в строительной механике [Текст] / А.И. Виноградов. - Харьков: Вища школа, 1973. - 168 с.

2. Ройтман М.И., Методы оптимального проектирования деформируемых тел [Текст] / М.И. Ройтман, Г.С. Шапиро. - М.: Наука, 1976. - 266 с.

3. Основы расчета и проектирования комбинированных и сталебетонных конструкций [Текст] / Э.Д. Чихладзе, Г.Л. Ватуля, Ю.П. Китов [и др.]; под ред. Э.Д. Чихладзе. - К.: Транспорт Украины, 2006. - 104 с.

Рецензент д-р техн. наук, профессор А.А. Плугин

Ватуля Гліб Леонідович, канд. техн. наук, доцент, заведуючий кафедрою будівельної механіки і гідравліки. Орел Євген Федорович, канд. техн. наук, доцент кафедри колії та колійного господарства. Левчук Світлана Валеріївна, слухач ІППК, гр. МЗ-ЗС-Б-11.

Андрущенко Ігор Миколайович, слухач ІППК, гр. МЗ-ЗС-Б-11.

Vatulya G.L. cand. of techn. sciences, Orel E.F., cand. of techn. sciences Levchyk S.V., Andrushenko S.M. 